\title{
JEAN-LUC STEUX
}

\section{Problème de Dirichlet pour un opérateur elliptique dans un domaine à point cuspide}

\author{
Annales de la faculté des sciences de Toulouse $6^{e}$ série, tome $6, \mathrm{n}^{\circ} 1$ \\ (1997), p. 143-175 \\ <http://www.numdam.org/item?id=AFST_1997_6_6_1_143_0>
}

(C) Université Paul Sabatier, 1997, tous droits réservés.

L'accès aux archives de la revue «Annales de la faculté des sciences de Toulouse » (http://picard.ups-tlse.fr/ annales/) implique l'accord avec les conditions générales d'utilisation (http://www.numdam.org/conditions). Toute utilisation commerciale ou impression systématique est constitutive d'une infraction pénale. Toute copie ou impression de ce fichier doit contenir la présente mention de copyright.

\section{NumDam}

Article numérisé dans le cadre du programme Numérisation de documents anciens mathématiques http://www.numdam.org/ 


\title{
Problème de Dirichlet pour un opérateur elliptique dans un domaine à point cuspide ${ }^{(*)}$
}

\author{
JEAN-LUC STEUX ${ }^{(1)}$
}

RÉSUMÉ. - Nous étudions la structure des solutions $u$ du problème de Dirichlet associé à un opérateur proprement elliptique sur un domaine $U$ dont le bord contient un point cuspide. Lorsque le second membre est $C^{\infty}$ jusqu'au bord de $U$, la structure de $u$ dépend des propriétés des équations des deux branches du bord de $U$. Nous montrerons en particulier que $u$ est $C^{\infty}$ jusqu'au bord si les équations sont $C^{\infty}$.

\begin{abstract}
We study the structure of the solutions $u$ of the Dirichlet problem associated to a property elliptic operator on a domain $U$, the boundary of which countains a cusp points. When the second member is $C^{\infty}$ up to the boundary of $U$, the structure of $u$ depends on the properties of the equations of the two branches of the boundary of $U$. We will show in particular that $U$ is $C^{\infty}$ up to the boundary if the equations are $C^{\infty}$.
\end{abstract}

\section{Introduction et hypothèses}

Soit $U$ un ouvert borné de $\mathbb{R}^{2}$ dont le bord $\partial U$ contient l'origine 0 . On suppose que $\partial U$ est $C^{\infty}$ sauf en 0 . Soit $L$ un opérateur proprement elliptique d'ordre $2 m$ à coefficients $C^{\infty}$ sur $\bar{U}$. Il est bien connu (cf. [1]) que le problème de Dirichlet associé à $L$

$$
L u=f, \quad u \in \stackrel{\circ}{H}^{m}(U),
$$

(*) Reçu le 3 janvier 1995

(1) Université de Nantes, Département de Mathématiques et d'Informatique, 2 chemin de la Houssinière, F-44072 Nantes Cedex 03 (France) 
est "régulier" sur tout domaine $V$ tel que $0 \notin \bar{V}, \forall k \in \mathbb{N}$,

$$
\text { si } f \in H^{k-m}(U) \text { alors } u \in H^{k+m}(U \cap V) \text {. }
$$

Par contre, si $0 \in \bar{V}$, le résultat ci-dessus ne subsiste pas en général.

Ce qui se passe au voisinage de 0 dépend de la géométrie de $U$. Supposons que l'on puisse choisir des coordonnées cartésiennes de $\mathbb{R}^{2}$ de façon que

$$
U \cap B(0, \varepsilon)=\left\{(x, y) \in B(0, \varepsilon) \mid \varphi_{1}(x)<y<\varphi_{2}(x)\right\}
$$

(où $B(0, \varepsilon)$ est la boule de centre 0 et de rayon $\varepsilon$ ),

$$
\left\{\begin{array}{l}
\left.\left.\varphi_{1}, \varphi_{2} \in C^{1}([0, \varepsilon]) \cap C^{\infty}(] 0, \varepsilon\right]\right) \\
\left.\left.\varphi_{1}<\varphi_{2} \text { sur }\right] 0, \varepsilon\right] \\
\varphi_{1}(0), \varphi_{2}(0)=0 \\
\varphi_{1}^{\prime}(0)=0 .
\end{array}\right.
$$

Le cas où $\varphi_{1}$ et $\varphi_{2}$ sont des équations de droites a été abondamment traité dans la littérature $: U$ coïncide alors localement avec un secteur plan (cf. [4] et les références qu'il y a dans [4]).

$\mathrm{Si}$, plus généralement,

$$
\left\{\begin{array}{l}
\varphi_{1}, \varphi_{2} \in C^{\infty}([0, \varepsilon]) \\
\varphi_{2}^{\prime}(0) \neq 0
\end{array}\right.
$$

alors $\varphi_{1}$ et $\varphi_{2}$ ne sont pas tangents en 0 et la régularité des solutions de $(0,1)$ est la même que pour le domaine tangent dont les équations des côtés sont $\Psi_{1}(x)=0, \Psi_{2}(x)=\varphi_{2}^{\prime}(0) x$ (cf. [2], [3]). Dans ce cas, l'angle $\omega$ tel que $\operatorname{tg} \omega=\varphi_{2}^{\prime}(0)$ intervient de façon déterminante. Par exemple si $L=\Delta$, on a pour la solution $u$ de $(0,1)$, si $k<\pi / \omega$ :

$$
\text { si } f \in H^{k-1}(U) \text { alors } u \in H^{k+1}(U) \text {. }
$$

Nous remarquons que pour cet exemple, plus l'angle $\omega$ est petit, plus le problème est régulier.

Notre sujet d'étude dans cet article est le cas où

$$
\varphi_{2}^{\prime}(0)=0,
$$

c'est-à-dire le cas d'un point cuspide saillant, ou pointe effilée. Nous supposons de plus que $\varphi:=\varphi_{2}-\varphi_{1}$ est continu sur $[0, \varepsilon]$ et que

$$
\forall k \in \mathbb{N}, \forall i=1,2, \ldots, \quad \varphi^{k} \varphi_{i}^{(k+1)} \longrightarrow 0 \text { quand } x \rightarrow 0
$$


Problème de Dirichlet pour un opérateur elliptique dans un domaine à point cuspide où $\varphi_{i}^{(j)}$ désigne la $j^{\text {ième }}$ dérivée de $\varphi_{i} ;$ pour $k=0,(0.6)$ redonne $\varphi_{1}^{\prime}(0)=0$, $\varphi_{2}^{\prime}(0)=0$.

DÉfINITION 0.1. - Nous regroupons l'ensemble des hypothèses (0.4), (0.5), (0.6) sous le nom d'hypothèse $\left(H_{0}\right)$ De plus, sous l'hypothèse $\left(H_{0}\right)$, nous poserons toujours $\varphi=\varphi_{2}-\varphi_{1}$.

Les résultats que nous avons obtenus sont à rapprocher de ceux donnés par Ibuki [6], Khelif [7], Mazya-Plamenevskii [8], Grisvard [5].

- Ibuki [6] s'est intéressé à des opérateurs d'ordre 2 mais avec des ouverts de la forme

$$
\left\{\left(x_{1}, x_{2}\right) \in \mathbb{R}^{2} \mid 0<x_{1}<d, 0<x_{2}<x_{1}^{\alpha}, \alpha>1\right\} .
$$

Il a donné des résultats avec un second membre dans $L^{2}$. Grisvard [5] a donné des résultats dans le même cadre pour le Laplacien et le problème de Stokes. Khelif [7] a donné des résultats concernant le Laplacien avec second membre dans $L^{2}$ mais pour des ouverts plus généraux que ceux étudiés par Ibuki. Ces ouverts sont très proches de ceux que nous avons étudiés et vérifient l'hypothèse $\left(\mathrm{H}_{0}\right)$.

- Les énoncés de Mazya-Plamenevskii [8] concernent en particulier des seconds membres dans des espaces $L^{p}$ à poids et pour un ouvert $U$ de $\mathbb{R}^{n}$ de révolution, de classe $C^{2}$ sauf en 0 et tel que

$$
U \cap B(0, \varepsilon)=\left\{x|| x^{\prime} \mid<\varphi\left(x_{n}\right), 0<x<\varepsilon\right\}
$$

pour $\varepsilon$ assez petit, avec $\varphi \in C^{2}\left(\left[0,1[), \varphi(0)=0, \varphi^{\prime}\left(x_{n}\right), \varphi\left(x_{n}\right)\right.\right.$, $\varphi^{\prime \prime}\left(x_{n}\right)$ tendant vers 0 quand $x_{n}$ tend vers 0 .

- Dans la note [9] nous avons donné des résultats concernant le Laplacien, des ouverts vérifiant $\left(\mathrm{H}_{0}\right)$ et des seconds membres $C^{\infty}$. Ici les opérateurs considérés sont d'ordre $2 m, m \geq 1$ et l'on obtient, sous certaines conditions (sect. 1), une régularité $C^{\infty}$.

\section{Notations}

- Si $q \in] 1,+\infty\left[, C_{q}([0, \varepsilon])\right.$ désigne l'espace des fonctions $\Psi$ de $C^{\infty}(] 0, \varepsilon[)$ telles qu'il existe une suite croissante $\left(q_{n}\right)_{n \geq 0}$ tendant vers $+\infty$ et des réels $a_{n}$ vérifiant

$$
\begin{aligned}
q_{0}=q, a_{0} \neq 0, \quad \Psi & -\sum_{0 \leq n \leq N} a_{n} x^{q_{n}}=\mathrm{O}\left(x^{q_{N+1}}\right) \\
- & 145-
\end{aligned}
$$




$$
\begin{aligned}
\Psi^{(j)}-\sum_{0 \leq n \leq N} a_{n} q_{n}\left(q_{n}-1\right) \cdots\left(q_{n}-j+1\right) x^{q_{n}-j}=\mathrm{O}\left(x^{q_{N+1}-j}\right), & \\
& \forall j \in \mathbb{N}^{*} \text { tel que } j \leq q_{N+1}, \forall N \in \mathbb{N} .
\end{aligned}
$$

- Si $q \in \mathbb{N} \backslash\{0,1\}, C_{q}^{\infty}([0, \varepsilon])=C^{\infty}([0, \varepsilon]) \cap C_{q}([0, \varepsilon])$

Voici quatre exemples fondamentaux de domaines à points cuspides vérifiant $\left(\mathrm{H}_{0}\right)$.

Exemple 1. $-\varphi_{1} \in C_{p_{1}}([0, \varepsilon]), \varphi_{2} \in C_{p_{2}}([0, \varepsilon])$ avec $\varphi>0$ et non plat en 0 .

Exemple 2.- $\varphi_{1} \in C_{p_{1}}^{\infty}([0, \varepsilon]), \varphi_{2} \in C_{p_{2}}^{\infty}([0, \varepsilon])$ avec $\varphi>0$, non plat en 0 et $2 \leq p_{2} \leq p_{1} \leq+\infty$ ( $\varphi_{1}$ et $\varphi_{2}$ sont par exemple des polynômes distincts de valuation $p_{1}$ et $\left.p_{2}\right)$.

Exemple 3. $-\varphi_{1}(x)=a_{1} x^{p_{1}} \sin x^{\beta_{1}}+b_{1} x^{p_{1}}, \varphi_{2}(x)=a_{2} x^{p_{2}} \sin x^{\beta_{2}}+$ $b_{2} x^{p_{2}}$ avec $p_{1}>p_{2}>1, \beta_{1}, \beta_{2} \leq 0, \beta=\inf \left(\beta_{1}, \beta_{2}\right)$ est tel que $\beta+p_{2}-1>0$, $a_{2}$ et $b_{2}$ vérifiant $\left|a_{2}\right|<\left|b_{2}\right|$.

Cet exemple ne vérifie pas les hypothèses nécessaires dans [4] et [7].

Exemple 4. $-\varphi_{1}(x)=C_{1} \mathrm{e}^{-\left(1 / x^{\alpha_{1}}\right)}, \varphi_{2}(x)=C_{2} \mathrm{e}^{-\left(1 / x^{\alpha_{2}}\right)}$ avec $\alpha_{1}$, $\alpha_{2}>0, C_{1} \neq C_{2}$ si $\alpha_{1}=\alpha_{2}$.

Cet exemple ne vérifie pas les hypothèses d'Ibuki [6].

En fait, quoique vérifiant tous $\left(\mathrm{H}_{0}\right)$, ces exemples n'ont pas les mêmes propriétés. Voici une suite d'hypothèses dont nous verrons à la section 2 qu'elles sont de plus en plus fortes.

- $\left(\mathrm{H}_{1}\right)$ désignera l'hypothèse suivante :

- l'hypothèse $\left(\mathrm{H}_{0}\right)$ est vérifiée,

$-\exists p_{0}>1, \exists C_{0}>0, \exists \varepsilon_{0}>0$ tels que $\forall x \in\left[0, \varepsilon_{0}\right], \varphi(x) \geq C_{0} x^{p_{0}}$.

Sous l'hypothèse $\left(\mathrm{H}_{1}\right)$, on se fixe $p \leq p_{0}, p$ choisi le plus petit possible tel que

$$
\begin{gathered}
\varphi(x) \geq C_{0} x^{p} . \\
-146-
\end{gathered}
$$


Problème de Dirichlet pour un opérateur elliptique dans un domaine à point cuspide

Evidemment on a $p>1$. Comme $\left|\varphi_{1}(x)\right| \leq C x$ sous l'hypothèse $\left(\mathrm{H}_{1}\right)$, on a

$$
\exists p_{0}^{\prime} \in[1, p] \text { tel que } \varphi(x) \geq C\left|\varphi_{1}(x)\right|^{p_{0}^{\prime}} .
$$

Toujours sous l'hypothèse $\left(\mathrm{H}_{1}\right)$, on se fixe $p^{\prime} \in\left[1, p_{0}^{\prime}\right]$ choisi "le plus petit possible" pour lequel

$$
\exists C^{\prime}>0 \text { tel que } \varphi(x) \geq C^{\prime}\left|\varphi_{1}(x)\right|^{p^{\prime}}, \quad \forall x \in[0, \varepsilon] .
$$

Seul l'exemple 4 ne vérifie pas l'hypothèse $\left(\mathrm{H}_{1}\right)$.

- Pour définir l'hypothèse $\left(\mathrm{H}_{2}\right)$ nous aurons besoin des notations suivantes : on pose $E_{0}=\mathbb{C}$ et pour $J$ dans $\mathbb{N}^{*}$ on note $E_{J}$ l'espace des combinaisons linéaires de fonctions de la forme

$$
\prod_{1 \leq k \leq K} \varphi^{n_{k}} \varphi_{i_{k}}^{\left(n_{k}+1\right)}
$$

avec

$$
i_{k} \in\{1,2\}, n_{k} \geq 0, \sum_{1 \leq k \leq K}\left(n_{k}+1\right)=J
$$

$\left(\mathrm{H}_{2}\right)$ désignera les hypothèses suivantes :

- l'hypothèse $\left(\mathrm{H}_{1}\right)$ est vérifiée,

- il existe une suite $(\delta(J))_{J \geq 1}$ positive, décroissante, telle que

$$
\sum_{J \geq 1} \delta(J)=+\infty
$$

et satisfaisant à

$$
\forall e \in E_{J}, \quad \varphi^{-\delta(1)-\cdots-\delta(J)} e \quad \text { est continue en } 0 \text {; }
$$

on peut toujours supposer que $\delta(J) \leq(p-1) / p$; on pose

$$
A(J)=\sum_{1 \leq K \leq J} \delta(K)
$$

- $\left(\mathrm{H}_{3}\right)$ désignera l'hypothèse : $\left(\varphi_{1}, \varphi_{2}\right)$ est du type de l'exemple 1 .

- $\left(\mathrm{H}_{4}\right)$ désignera l'hypothèse : $\left(\varphi_{1}, \varphi_{2}\right)$ est du type de l'exemple 2 . 
Voici successivement les hypothèses sur l'opérateur $L$ et quelques espaces fonctionnels utiles.

Hypothèse $(\mathcal{L})$

- $L\left(x, y ; \partial_{x}, \partial_{y}\right)=\sum_{|\alpha| \leq 2 m} a_{\alpha} \partial^{\alpha}$ avec $a_{\alpha} \in C^{\infty}(\bar{U})$;

- $L$ est proprement elliptique;

- soit $L_{0}\left(\partial_{x}, \partial_{y}\right)$ la partie principale de $L$ gelée en $(0,0) ; \theta$ désignera par la suite la variable homogénéisée $\left(y-\varphi_{1}\right) \varphi^{-1}$; on est amené à utiliser

$$
L_{0}\left(\frac{\lambda}{i}, \mathrm{~d}_{\theta}\right)=\sum_{|\alpha|=2 m} a_{\alpha}(0,0)\left(\frac{\lambda}{i}\right)^{\alpha_{1}} \mathrm{~d}_{\theta}^{\alpha_{2}}
$$

et on suppose que

$$
\forall \lambda \in \mathbb{R}, \quad L_{0}\left(\frac{\lambda}{i}, \mathrm{~d}_{\theta}\right) \text { est injectif sur } \stackrel{\circ}{H}^{m}(] 0,1[) .
$$

On peut montrer que si $\lambda=0, L_{0}\left(0, \mathrm{~d}_{\theta}\right)$ est toujours injectif sur $\stackrel{\circ}{H}^{m}$ (] 0,1 [). C'est le cas aussi pour $\lambda$ quelconque dans $\mathbb{R}$ et $m=1$ lorsque les deux premières conditions sont vérifiées. Ce n'est pas toujours le cas par contre si $m=2$. Ceci sera développé dans le paragraphe 2.2 .

Définition 0.2.- $C^{\infty}(\bar{U})=\left\{u \in C^{\infty}(U)\right.$ telles que $\forall \alpha \partial^{\alpha} u$ se prolonge en une fonction continue sur $\bar{U}\}$.

Si $q \in \mathbb{N} \cup\{+\infty\}, C_{q}^{\infty}(\bar{U})=\left\{u \in C^{\infty}(U)\right.$ telles que $\partial^{\alpha} u(0,0)=0, \forall \alpha$ tel que $|\alpha|<q\}$.

Si $n \in \mathbb{N}, V^{n}(U)=\left\{u \in \mathcal{D}^{\prime}(U)\right.$ telles que $\varphi^{|\alpha|-n} \partial^{\alpha} u \in L^{2}(U), \forall \alpha$ tel que $|\alpha| \leq n\}$.

$W^{n, \infty}(U)$ est l'espace des fonctions dont toutes les dérivées jusqu'à l'ordre $n$ sont bornées sur $U$. 
Problème de Dirichlet pour un opérateur elliptique dans un domaine à point cuspide

\section{Résultats}

\subsection{Cas du second membre plat}

THÉORÈME 1.1. - Soient $\left(\varphi_{1}, \varphi_{2}\right)$ vérifiant l'hypothèse $\left(H_{0}\right), L$ vérifiant l'hypothèse $(\mathcal{L})$ et soit $n \in \mathbb{N}$. Si $f \in V^{n}(U)$, alors $u$ solution $d u$ problème (0.1) appartient $\grave{a} V^{n+2 m}(U)$.

La démontration de ce résultat est une réécriture plus simple (car on se trouve dans un cadre plus simple) des résultats de Mazya-Plamenevskii [8]. Le théorème 1.1 donne en particulier que pour $L=\Delta$, si $f \in L^{2}(U)$ alors $u \in V^{2}(U)$, donc $u \in H^{2}(U)$. (On retrouve pour les opérateurs considérés dans ces articles les propriétés données dans [4], [5], [6] et [7].)

Corollaire 1.2. - Sous les mêmes hypothèses sur $\left(\varphi_{1}, \varphi_{2}\right)$ et $L$ que dans le théorème 1.1, les vecteurs propres de l'opérateur $L$ sont dans $C_{\infty}^{\infty}(\bar{U})$.

Ce n'est pas le cas en général pour les polygones curvilignes dont les tangentes aux points singuliers forment un angle $\omega$ différent de 0 (cf. [3] où nous avons cherché à caractériser de tels polygones curvilignes $G$ pour lesquels les vecteurs propres du problème de Dirichlet pour le laplacien sur $G$ sont $C^{\infty}$ jusqu'au bord de $G$ ).

THÉORÈME 1.3.-Si $\left(\varphi_{1}, \varphi_{2}\right)$ vérifie l'hypothèse $\left(H_{1}\right)$, si L vérifie l'hypothèse $(\mathcal{L})$ et si $f \in C_{\infty}^{\infty}(\bar{U})$, alors $u \in C_{\infty}^{\infty}(\bar{U})$.

\subsection{Cas du second membre $C^{\infty}$}

L'un des résultats les plus marquants est que, lorsque les côtés ont des équations $C^{\infty}$, le problème de Dirichlet a la régularité $C^{\infty}$.

THÉORÈME 1.4. - Si $\left(\varphi_{1}, \varphi_{2}\right)$ vérifie l'hypothèse $\left(H_{4}\right)$ et si $L$ vérifie l'hypothèse $(\mathcal{L})$, alors si $f \in C^{\infty}(\bar{U})$, u solution de $(0.1)$ est $C^{\infty}(\bar{U})$.

Lorsque l'hypothèse $\left(\mathrm{H}_{4}\right)$ n'est pas vérifiée, il apparaît des singularités. 
Lemme 1.5. - Soit $\left(\varphi_{1}, \varphi_{2}\right)$ vérifiant $\left(H_{2}\right), L$ l'hypothèse $(\mathcal{L})$ et soit $\alpha \in \mathbb{N}^{2}$. Alors pour tout $n \in \mathbb{N}^{*}$, il existe $\sigma_{\alpha, n}$ tel que

$$
L\left(\chi \sigma_{\alpha, n}\right)-x^{\alpha_{1}} y^{\alpha_{2}} \in V^{n}(U), \quad \chi \sigma_{\alpha, n} \in \stackrel{\circ}{H}^{m}(U)
$$

ò̀ $\chi \in C^{\infty}\left(\mathbb{R}^{2}\right)$ est à support dans la boule $B(0, \varepsilon)$, vaut 1 au voisinage de 0 ,

$$
\sigma_{\alpha, n} \in \mathcal{S}^{\alpha_{1} / p+\alpha_{2} / p^{\prime}+2 m}
$$

où pour s réel l'espace $\mathcal{S}^{s}$ est introduit dans la définition 4.1. Par exemple $\sigma_{0, n}$ est une combinaison linéaire d'éléments de la forme $x^{i} y^{j} \varphi_{1}^{k}\left(y-\varphi_{1}\right)^{a}$ avec $i, j, k, a \in \mathbb{N}, i / p+j+k / p^{\prime}+a \geq 2 m$.

THÉORÈME 1.6. - Sous l'hypothèse $\left(H_{2}\right)$ et $(\mathcal{L})$ pour $L$, si $f \in C^{\infty}(\bar{U})$, $\forall n \in \mathbb{N}^{*}$, on pose :

$$
S_{n}=\chi \sum_{|\alpha| \leq[p n]-1} \frac{1}{\alpha !}\left(\partial^{\alpha} f\right)(0,0) \sigma_{\alpha, n} .
$$

Alors u solution dans $\stackrel{\circ}{H}^{m}(U)$ du problème (0.1) vérifie

$$
u-S_{n} \in V^{n+2 m}(U) \text {. }
$$

Autrement dit les $S_{n}$ constituent un développement asymptotique de u en 0 .

THÉORÈME 1.7.- Sous les hypothèses $\left(H_{2}\right)$ et $(\mathcal{L})$, si $f$ appartient à $C_{q}^{\infty}(\bar{U}), q \geq 0$, alors $u$ solution du problème (0.1) appartient à $C^{2 m+(q / p)}(\bar{U})$ si $2 m+(q / p)$ n'est pas entier et $u$ appartient $\grave{a}$ $W^{2 m+(q / p), \infty}(U)$ si $2 m+(q / p)$ est entier.

Pour mieux décrire la régularité sous l'hypothèse $\left(\mathrm{H}_{3}\right)$ nous introduisons la définition suivante.

DÉfinition 1.8.- Si $\sigma \in] 0,1\left[\right.$, on appelle $C^{\sigma, 0}(\bar{U})$ l'espace des fonctions u telles que

$$
\sup _{\substack{x_{1} \neq x_{2} \\\left(x_{1}, y_{1}\right),\left(x_{2}, y_{1}\right) \in \bar{U}}}\left|u\left(x_{1}, y_{1}\right)-u\left(x_{2}, y_{1}\right)\right|\left|x_{1}-x_{2}\right|^{-\sigma}
$$

est borné indépendamment de $y_{1}$. De même $C^{0, \sigma}(\bar{U})$ est l'espace des fonctions u telles que

$$
\sup _{y_{1} \neq y_{2}}\left|u\left(x_{1}, y_{1}\right)-u\left(x_{1}, y_{2}\right)\right|\left|y_{1}-y_{2}\right|^{-\sigma}
$$

est borné indépendamment de $x_{1}$. 
Problème de Dirichlet pour un opérateur elliptique dans un domaine à point cuspide

Si $\sigma=0$, on note $C^{0,0}(\bar{U})$ l'espace $C^{0}(\bar{U})$. Si s et $s^{\prime}$ sont deux réels positifs, on définit $C^{s, s^{\prime}}$ par:

$$
\begin{gathered}
u \in C^{s, s^{\prime}} \text { si et seulement si } \partial_{x}^{\alpha} \partial_{y}^{\beta} u \in C^{\sigma, 0}(\bar{U}) \cap C^{0, \tau}(\bar{U}) \\
\forall \alpha, \beta \in \mathbb{N}, \quad \forall \sigma, \tau \in\left[0,1\left[\text { tels que } \frac{\alpha+\sigma}{s}+\frac{\beta+\tau}{s^{\prime}} \leq 1 .\right.\right.
\end{gathered}
$$

THÉORÈmE 1.9. - Sous l'hypothèse $\left(H_{3}\right)$ et $(\mathcal{L})$ pour $L$, si $f$ appartient $\grave{a} C_{q}^{\infty}(\bar{U}), q \geq 0$, alors $u$ solution du problème (0.1) appartient $\grave{a}$ $C^{a(q / p)+2 m,(q / p)+2 m}(\bar{U})$ ò̀ $a=p /\left(p-p_{2}+1\right)$.

Ces résultats s'appliquent au laplacien (et en général à tous les opérateurs proprement elliptiques d'ordre deux), au biplacien. Ils peuvent aussi s'appliquer au problème de Stokes sous certaines conditions pour les données (ceci sera développé à la fin du paragraphe 2.2).

\subsection{Grandes lignes de la démonstration}

Les énoncés des théorèmes 1.3 et corollaires 1.2 découlent du théorème 1.1. Pour obtenir le théorème 1.1 on se ramène à un problème de Dirichlet sur la bande ] 0,1 [ $\times \mathbb{R}$ en faisant le changement de variable

$$
(x, y) \longrightarrow(\theta, t)
$$

où

$$
\theta=\frac{y-\varphi_{1}}{\varphi}, \quad t=-\int_{x}^{+\infty} \frac{\mathrm{d} \sigma}{\varphi(\sigma)}
$$

où $\varphi$ est prolongé de façon que $1 / \varphi \in L^{1}(] \varepsilon,+\infty[)$. Cette méthode est reprise de [8].

Lorsque $f \in C^{\infty}(\bar{U})$, on retranche une partie polynomiale $f_{n}$ telle que $f-f_{n} \in V^{n}(U)$. Ensuite on construit une fonction $u_{n}\left(f_{n}\right)$ de $\stackrel{\circ}{H}^{m}(U)$ telle que :

$$
L u_{n}=f_{n}+r_{n}
$$

avec $r_{n} \in V^{n}(U)$ (résolution polynomiale approchée). On a alors

$$
L\left(u-u_{n}\right)=f-f_{n}-r_{n}
$$

avec $u-u_{n} \in \stackrel{\circ}{H}^{m}(U)$. Les résultats précédents donnent alors que

$$
u-u_{n} \in V^{n+2 m}(U) \text {. }
$$


La résolution polynomiale approchée est obtenue à l'aide du changement de variables $\mathcal{C}$ suivant :

$$
(x, y) \longrightarrow(X, Y)
$$

où

$$
X=x, \quad Y=y-\varphi_{1}(x) .
$$

Le plan est le suivant : dans le paragraphe 2.1 on donne certaines propriétés vérifiées par les domaines, dans 2.2 on étudie des exemples d'opérateurs et dans 2.3 on étudie les changements de variables (1.2) et (1.3). Dans la section 3, on démontre les théorèmes et corollaires 1.1 à 1.3. Ensuite dans la section 4, on étudie la résolution polynomiale approchée et on en déduit les énoncés 1.4 à 1.9 .

\section{Analyse fonctionnelle}

\subsection{Propriétés vérifiées par les domaines}

Proposition 2.1.- Si $\left(\varphi_{1}, \varphi_{2}\right)$ vérifie l'hypothèse $\left(H_{3}\right)$, alors $\left(\varphi_{1}, \varphi_{2}\right)$ vérifie l'hypothèse $\left(H_{2}\right)$ avec $\delta(N)=\left(p_{2}-1\right) / p$. De plus $p^{\prime}=\sup \left(1, p / p_{1}\right)$.

\section{Démonstration}

a) Pour $i=1,2$ il existe une suite croissante $\left(q_{n, i}\right)_{n \geq 0}$ tendant vers $+\infty$ et des réels $a_{n, i}$ tels que

$$
\begin{aligned}
& q_{0, i}=p_{i}, a_{0, i} \neq 0, \\
& \varphi_{i}-\sum_{0 \leq n \leq N} a_{n, i} x^{q_{n, i}}=\mathrm{O}\left(x^{q_{N+1, i}}\right) \\
& \varphi_{i}^{(j)}-\sum_{0 \leq n \leq N} a_{n, i} q_{n, i}\left(q_{n, i}-1\right) \cdots\left(q_{n, i}-j+1\right) x^{q_{n, i}-j}=\mathrm{O}\left(x^{q_{N+1, i}-j}\right)
\end{aligned}
$$

pour $j$ tel que $1 \leq j \leq q_{N+1, i}$. Alors si $p_{2}<p_{1}$ en ordonnant $\left\{q_{n, i}, i=1,2\right\}$ on obtient une suite croissante $\left(q_{n, 3}\right)_{n \geq 0}$ tendant vers $+\infty$. Posons :

$$
a_{n, 3}= \begin{cases}a_{n, 2}-a_{n, 1} & \text { si } q_{n, 1}=q_{n, 2}=q_{n, 3} \\ a_{n, 2} & \text { si } q_{n, 2}=q_{n, 3} \neq q_{n, 1} \\ -a_{n, 1} & \text { si } q_{n, 1}=q_{n, 3} \neq q_{n, 2}\end{cases}
$$


Problème de Dirichlet pour un opérateur elliptique dans un domaine à point cuspide

On a $q_{0,3}=p_{2}, a_{0,3}=a_{0,2} \neq 0$ et $\varphi$ vérifie (2.1) avec $i=3$. On a alors $p=p_{2}$.

- Si $p_{1}=p_{2}$ avec $a_{0,1} \neq a_{0,2}$ en ordonnant encore $\left\{q_{n, i}, i=1,2\right\}$, on obtient une suite croissante $\left(q_{n, 3}\right)_{n \geq 0}$ tendant vers $+\infty$ et des réels $a_{n, 3}$ tels que l'on ait :

$$
q_{0,3}=p_{1}, \quad a_{0,3}=a_{0,2}-a_{0,1} \neq 0
$$

et $\varphi$ vérifie (2.1) avec $i=3$. On a alors $p=p_{2}$.

- Si $p_{1}=p_{2}$ avec $a_{0,1}=a_{0,2}$, on pose

$$
p=\inf \left\{q_{n, i}, i=1,2 \mid a_{n, 2}-a_{n, 1} \neq 0\right\} .
$$

Alors si on ordonne $\left\{q_{n, i}>p, i=1,2\right\}$, on obtient la suite $\left(q_{n, 3}\right)_{n \geq 0}$ et les réels $a_{n, 3}$ tels que

$$
q_{0,3}=p, \quad a_{0,3} \neq 0
$$

et $\varphi$ vérifie $(2.1)$

b) D'après ce qui précède, comme $p_{2} \leq p_{1}$

$$
e=\prod_{1 \leq k \leq K} \varphi^{n_{k}} \varphi_{i_{k}}^{\left(n_{k}+1\right)} \text { avec } \sum_{1 \leq k \leq K}\left(n_{k}+1\right)=J
$$

est égal à

$$
x^{n_{1} p+p_{2}-n_{1}-1+\cdots+n_{k} p+p_{2}-n_{k}-1}\left(C+\mathrm{O}\left(x^{\varepsilon}\right)\right)
$$

où $\varepsilon \geq 0$. Comme $p \geq p_{2}$

$$
e=x^{\left(p_{2}-1\right) J+\varepsilon^{\prime}}\left(C+\mathrm{O}\left(x^{\varepsilon}\right)\right)
$$

où $\varepsilon^{\prime} \geq 0$. Ainsi le produit de $\varphi^{-A(J)}$ par $e$ est continu au voisinage de 0 si $-A(J) p+\left(p_{2}-1\right) J \geq 0$. On posera dans ce cas

$$
A(J)=\frac{p_{2}-1}{p} J .
$$

Il est immédiat d'après la définition de $p^{\prime}$ que $p^{\prime}=\sup \left(1, p / p_{1}\right)$.

Proposition 2.2.- Si $\left(\varphi_{1}, \varphi_{2}\right)$ est du type de l'exemple 3, alors $\left(\varphi_{1}, \varphi_{2}\right)$ vérifie l'hypothèse $\left(H_{2}\right)$ avec $p=p_{2}, \delta(J)=(p+\beta-1) / p$. De plus $p^{\prime}=1$. 
Proposition 2.3.- Si $\varphi_{1} \in C_{p_{1}}([0, \varepsilon])$ et si

$$
\varphi_{2}(x)=a_{2} x^{p_{2}} \sin x^{\beta}+b_{2} x^{p_{2}}
$$

avec

$$
p_{1}>p_{2}>1, \quad 1-p_{2}<\alpha_{2}<0, \quad 0<a_{2}<b_{2} \quad \text { ou } \quad b_{2}<a_{2}<0,
$$

alors $\left(\varphi_{1}, \varphi_{2}\right)$ vérifie l'hypothèse $\left(H_{2}\right)$ avec $p=p_{2}, \delta(J)=(p+\beta-1) / p$. De plus on a encore $p^{\prime}=1$.

\subsection{Exemples d'opérateurs}

Remarque 2.4. - Pour tout opérateur tel que $a_{2 m, 0}(0,0) \neq 0$,

$$
L_{0}\left(0, \mathrm{~d}_{\theta}\right)=a_{2 m, 0}(0,0) \mathrm{d}_{\theta}^{2 m}
$$

est injectif sur $\stackrel{\circ}{H}^{m}(] 0,1[)$. Pour le démontrer on regarde les polynômes de la forme

$$
\alpha_{2 m-1} \theta^{2 m-1}+\cdots+\alpha_{0}
$$

qui s'annulent ainsi que leurs dérivées jusqu'à l'ordre $m-1$ en 0 et en 1 . On obtient un système dont le déterminant est non nul, d'où la seule solution 0 .

Proposition 2.5. - Pour tout opérateur proprement elliptique d'ordre 2, pour $\lambda$ dans $\mathbb{R}, L_{0}\left(\lambda / i, \mathrm{~d}_{\theta}\right)$ est injectif sur $\stackrel{\circ}{H}^{1}(] 0,1[)$.

Démonstration. - En utilisant la définition de l'ellipticité propre de l'opérateur $L$, on obtient que pour tout $\lambda \neq 0$ l'équation caractéristique de l'opérateur $L_{0}\left(\lambda / i, \mathrm{~d}_{\theta}\right)$

$$
a_{0,2}(0,0) r^{2}-a_{1,1}(0,0) i \lambda r-a_{2,0}(0,0) \lambda^{2}
$$

a une racine à partie réelle $>0$ et une à partie réelle $<0$. Si on désigne par $r_{1}$ et $r_{2}$ les racines de $(2.2)$, les éléments du noyau de $L_{0}\left(\lambda / i, \mathrm{~d}_{\theta}\right)$ sont de la forme

$$
a e^{r_{1} \theta}+b e^{r_{2} \theta} .
$$

D'après ce qui précède, comme $r_{1}-r_{2}$ ne peut être imaginaire pur, on a $e^{r_{1}} \neq e^{r_{2}}$; donc la seule fonction de la forme (2.3) qui s'annule pour $\theta=0$ et $\theta=1$ est la fonction nulle. 
Problème de Dirichlet pour un opérateur elliptique dans un domaine à point cuspide

Proposition 2.6. - Pour un opéateur proprement elliptique d'ordre 4 dont le symbole en 0

$$
L_{0}\left(\xi_{1}, \xi_{2}\right)=\sum_{|\alpha|=4} a_{\alpha}(0,0) \xi_{1}^{\alpha_{1}} \xi_{2}^{\alpha_{2}}
$$

peut se mettre sous la forme

$$
a_{0,4}(0,0)\left(\alpha_{1} \xi_{1}-\xi_{2}\right)\left(\bar{\alpha}_{1} \xi_{1}-\xi_{2}\right)\left(\alpha_{2} \xi_{1}-\xi_{2}\right)\left(\bar{\alpha}_{2} \xi_{1}-\xi_{2}\right)
$$

avec $\alpha_{1}$ et $\alpha_{2}$ imaginaires purs, $L_{0}\left(\lambda / i, \mathrm{~d}_{\theta}\right)$ est injectif sur $\stackrel{\circ}{H}^{2}(] 0,1[)$.

Démonstration. - D'après l'hypothèse

$$
a_{4,0}(0,0)+\cdots+a_{0,4}(0,0) \tau^{4}
$$

peut se mettre sous la forme

$$
a_{0,4}(0,0)\left(\alpha_{1}-\tau\right)\left(\bar{\alpha}_{1}-\tau\right)\left(\alpha_{2}-\tau\right)\left(\bar{\alpha}_{2}-\tau\right)
$$

Les racines du polynôme caractéristique de $L_{\theta}\left(\lambda / i, \mathrm{~d}_{\theta}\right)$ sont alors $i \lambda \alpha_{1}$, $i \lambda \bar{\alpha}_{1}, i \lambda \alpha_{2}, i \lambda \bar{\alpha}_{2}$.

- Cas où il y a racine double (non nulle). On pose $i \lambda \alpha_{1}=\lambda \beta, \beta \in \mathbb{R}^{*}$. L'autre racine est alors $-\lambda \beta$. La solution générale de $L_{0}\left(\lambda, \mathrm{d}_{\theta}\right) u=0$ est alors

$$
u=a_{1} e^{\lambda \theta \beta}+a_{2} e^{-\lambda \theta \beta}+a_{3} \theta e^{\lambda \theta \beta}+a_{4} \theta e^{-\lambda \theta \beta} .
$$

$u(0)=u(1)=u^{\prime}(0)=u^{\prime}(1)=0$ est équivalent à :

$$
\left\{\begin{array}{l}
a_{1}+a_{2}=0 \\
a_{1} e^{\lambda \beta}+a_{2} e^{-\lambda \beta}+a_{3} e^{\lambda \beta}+a_{4} e^{-\lambda \beta}=0 \\
a_{1} \lambda \beta-a_{2} \lambda \beta+a_{3}+a_{4}=0 \\
a_{1} \lambda \beta e^{\lambda \beta}-a_{2} \lambda \beta e^{-\lambda \beta}+a_{3}(1+\lambda \beta) e^{\lambda \beta}+a_{4}(1-\lambda \beta) e^{-\lambda \beta}=0 .
\end{array}\right.
$$


Le déterminant de ce système (où $a_{1}, a_{2}, a_{3}, a_{4}$ sont les inconnues) vaut $(\lambda \beta)^{2}-\left(e^{\lambda \beta}-e^{-\lambda \beta}\right)^{2}$. Mais

$$
4(\operatorname{sh} \lambda \beta)^{2}=\lambda^{2} \beta^{2}, \quad \beta \in \mathbb{R}^{*}
$$

n'a pas de solution. L'opérateur $L_{0}\left(\lambda / i, \mathrm{~d}_{\theta}\right)$ est donc injectif sur $\stackrel{\circ}{H}^{2}(] 0,1[)$.

- Cas où il n'y a pas de racines doubles (c'est-à-dire on a 4 racines distinctes). Les racines peuvent dans ce cas s'écrire $\lambda \beta_{1},-\lambda \beta_{1}, \lambda \beta_{2}$, $-\lambda \beta_{2}$, avec par exemple $\beta_{1}>0, \beta_{2}<0,\left|\beta_{2}\right|<\left|\beta_{1}\right|$. Rechercher la solution générale de $L_{0}\left(\lambda, \mathrm{d}_{\theta}\right) u=0$ qui s'annule ainsi que sa dérivée en 0 et en 1 revient dans ce cas à chercher les solutions du système :

$$
\left\{\begin{array}{l}
a_{1}+a_{2}+a_{3}+a_{4}=0 \\
a_{1} e^{\lambda \beta_{1}}+a_{2} e^{-\lambda \beta_{1}}+a_{3} e^{\lambda \beta_{2}}+a_{4} e^{-\lambda \beta_{2}}=0 \\
a_{1} \lambda \beta_{1}-a_{2} \lambda \beta_{1}+a_{3} \lambda \beta_{2}-a_{4} \lambda \beta_{2}=0 \\
a_{1} \lambda \beta_{1} e^{\lambda \beta_{1}}-a_{2} \lambda \beta_{1} e^{-\lambda \beta_{1}}+a_{3} \lambda \beta_{2} e^{\lambda \beta_{2}}-a_{4} \lambda \beta_{2} e^{-\lambda \beta_{2}}=0 .
\end{array}\right.
$$

Le déterminant de ce système vaut en posant

$$
\begin{gathered}
\lambda\left(\beta_{1}-\beta_{2}\right)=x, \quad \lambda\left(\beta_{1}+\beta_{2}\right)=y . \\
\Delta=x^{2}(\operatorname{ch} y-1)-y^{2}(\operatorname{ch} x-1)
\end{gathered}
$$

d'où

$$
\Delta=0 \Longleftrightarrow x^{2} y^{2}\left(\frac{1}{2 !}+\frac{y^{2}}{4 !}+\cdots-\left(\frac{1}{2 !}+\frac{x^{2}}{2 !}+\cdots\right)\right)=0
$$

Comme la fonction $x \rightarrow 1 /(2 !)+x^{2} /(4 !)+\cdots$ est strictement croissante et que $x>0$, on a $\Delta \neq 0$. Ainsi, l'opérateur $L_{0}\left(\lambda, \mathrm{d}_{\theta}\right)$ est injectif sur $\stackrel{\circ}{H}^{2}(] 0,1[)$.

- Le cas $\lambda=0$ a été étudié dans la remarque 2.4 .

Remarque 2.7. - Dans le cas où $\alpha_{1}$ et $\alpha_{2}$ ne sont pas imaginaires purs, l'opérateur $L_{0}\left(\lambda / i, \mathrm{~d}_{\theta}\right)$ est non injectif si et seulement si l'équation (2.4) avec $\beta$ complexe (non nécessairement réel) a des solutions. Mais l'étude de $\operatorname{sh} \lambda \beta=2 \lambda \beta$ revient en posant $\beta=a+i b$ au système

$$
\left\{\begin{array}{l}
\cos \lambda b \operatorname{sh} \lambda a=2 \lambda a \\
\sin \lambda b \operatorname{ch} \lambda a=2 \lambda b .
\end{array}\right.
$$

Ce système ayant des solutions, il existe des opérateurs d'ordre 4 pour lesquels $L_{0}\left(\lambda / i, \mathrm{~d}_{\theta}\right)$ n'est pas injectif sur $\stackrel{\circ}{H}^{m}(] 0,1[)$. 
Problème de Dirichlet pour un opérateur elliptique dans un domaine à point cuspide

Proposition 2.8. - Le laplacien et le biplacien vérifient l'hypothèse $(\mathcal{L})$. Le problème de Stokes : étude de $v_{1}, v_{2}$ et $p$ solutions du système

$$
\begin{cases}-\triangle v_{1}+\frac{\partial p}{\partial x}=f_{1} & \\ -\Delta v_{2}+\frac{\partial p}{\partial y}=f_{2} & \\ \frac{\partial v_{1}}{\partial x}+\frac{\partial v_{2}}{\partial y}=0 & \text { dans } U \\ v_{1}=v_{2}=0 & \text { sur } \partial U\end{cases}
$$

avec $f_{1}, f_{2}$ dans $V^{n}(U), n \geq 1$, ou dans $C_{q}^{\infty}(\bar{U}), q \geq 0$, peut être transformé en l'étude de u tel que

$$
\left\{\begin{array}{l}
\triangle^{2} u=\frac{\partial f_{1}}{\partial y}-\frac{\partial f_{2}}{\partial x} \\
u \in \stackrel{\circ}{H}^{2}(U)
\end{array}\right.
$$

avec $\partial f_{1} / \partial y-\partial f_{2} / \partial x$ appartenant $\grave{a} V^{n-1}(U)$ ou à $C_{q-1}^{\infty}(\bar{U}), q \geq 1$.

Démonstration. - Le laplacien et le biplacien vérifient les conditions de la proposition 2.6 , d'où l'hypothèse $(\mathcal{L})$. Soient $v_{1}, v_{2}, p, f_{1}, f_{2}$ vérifiant (2.5) avec $f_{1}, f_{2} \in V^{n}(U)$ ou $C_{q}^{\infty}(\bar{U}), q \geq 0$. On peut suivre la méthode donnée par Grisvard [4] pour réduire (2.5) au système (2.6).

\section{3 Étude des changements de variables (1.2) et (1.3)}

\section{Changement de variable $\mathcal{C}$}

Le changement de variable $\mathcal{C}$ (cf. (1.3)), consiste à aplatir le côté $y=$ $\varphi_{1}(x)$. Au voisinage de 0

$$
G=\mathcal{C}(U)=\left\{(X, Y) \in \mathbb{R}^{2} \mid 0<Y<\varphi(X)\right\} .
$$

DÉfinition 2.9.- Si $n, \lambda \in \mathbb{N}$, soit $\mathcal{C}_{n, \lambda}$ les combinaisons linéaires d'expressions de la forme

$$
\varphi_{1}^{\left(n_{1}+1\right)} \cdots \varphi_{1}^{\left(n_{k}+1\right)}
$$

avec $n_{1}, \ldots, n_{k} \geq 0, n_{1}+\cdots+n_{k} \leq n, k \in \mathbb{N}^{*}, k \leq \lambda$. 
Remarque 2.10. $-\forall n \in \mathbb{N}$, si $S \in \mathcal{C}_{n, \lambda}$ alors $\varphi^{n} S$ tend vers 0 quand $x$ tend vers 0 .

Proposition 2.11. - Soit $\alpha=\left(\alpha_{1}, \alpha_{2}\right)$ un multi-indice. Alors

$$
\partial_{x, y}^{\alpha} u=\partial_{X, Y}^{\alpha} \tilde{u}+\sum_{|\beta| \leq|\alpha|} S_{\alpha, \beta} \partial_{X, Y}^{\beta} \tilde{u}
$$

ò̀ $\tilde{u}=u \circ \mathcal{C}^{-1}$ avec $S_{\alpha, \beta} \in \mathcal{C}_{|\alpha|-|\beta|,|\alpha|}$.

Démonstration. - On montre par récurrence que $\forall \alpha \in \mathbb{N}^{2}$,

$$
\partial_{x, y}^{\alpha} u=\sum_{|\beta| \leq|\alpha|} b_{\alpha, \beta} \partial_{X, Y}^{\beta} \widetilde{u}
$$

avec $b_{\alpha, \beta}=1$ si $\alpha=\beta, b_{\alpha, \beta} \in \mathcal{C}_{|\alpha|-|\beta|,|\alpha|}$ sinon. Pour cela on utilise que si $S \in \mathcal{C}_{n, \lambda}$, alors $\partial_{X} S \in \mathcal{C}_{n+1, \lambda}$.

Corollaire 2.12. - Soit

$$
L u=\sum_{|\beta| \leq 2 m} b_{\beta} \partial_{X, Y}^{\beta} \tilde{u}
$$

avec $\forall \beta, b_{\beta}=a_{\beta}+\sum_{|\beta| \leq|\alpha| \leq 2 m} S_{\alpha, \beta} a_{\alpha}$.

DÉfinition 2.13. - Si $a \in \mathbb{R}$ et $n \in \mathbb{N}$,

$$
\begin{aligned}
& u \in V_{a}^{n}(U) \quad \Longleftrightarrow \quad \forall|\alpha| \leq n, \varphi^{a+|\alpha|-n} \partial_{x, y}^{\alpha} u \in L^{2}(U), \\
& u \in V_{a}^{n}(G) \quad \Longleftrightarrow \quad \forall|\alpha| \leq n, \varphi^{a+|\alpha|-n} \partial_{x, y}^{\alpha} u \in L^{2}(G) .
\end{aligned}
$$

Corollaire 2.14. - Soit

$$
u \in V_{a}^{n} U \Longleftrightarrow \widetilde{u}=u \circ \mathcal{C}^{-1} \in V_{a}^{n}(G) .
$$

Ce corollaire est une conséquence de la remarque 2.10 et de la proposition 2.11. 
Problème de Dirichlet pour un opérateur elliptique dans un domaine à point cuspide

II. Changement de variable $\mathcal{T}$

On appelle $\mathcal{T}$ le changement de variable suivant

$$
(X, Y) \rightarrow(\theta, t), \quad \theta=\frac{Y}{\varphi}, t=-\int_{X}^{+\infty} \frac{\mathrm{d} \sigma}{\varphi(\sigma)} .
$$

On pose $\Omega=\mathcal{T}(G)=\mathcal{T} \circ \mathcal{C}(U)$

$$
v(\theta, t)=\left(u \circ \mathcal{C}^{-1} \circ \mathcal{T}^{-1}\right)(\theta, t)
$$

DÉFInITION 2.15. - Soit $F_{0}$ l'espace engendré par les produits d'un élément de la forme

$$
e x^{\alpha_{1}} \varphi^{\alpha_{2}} \theta^{\alpha_{3}} \varphi_{1}^{\alpha_{4}}
$$

et d'un élément de $C^{\infty}(\bar{U})$ où $\alpha_{1}, \alpha_{2}, \alpha_{3}, \alpha_{4} \in \mathbb{N}, e \in E_{J}, J \in \mathbb{N}$ avec $\alpha_{1}+\alpha_{2}+\alpha_{4}+J \geq 1$.

Remarque 2.16. - Les éléments de $F_{0}$ tendent vers 0 quand $x$ tend vers 0 .

Proposition 2.17. - Pour tout multi-indice $\alpha$ on a

$$
\partial_{x, y}^{\alpha} \tilde{u}=\varphi^{-|\alpha|}\left(\partial_{t, \theta}^{\alpha} v+\sum_{|\beta| \leq|\alpha|} b_{\alpha, \beta} \partial_{t, \theta}^{\beta} v\right)
$$

où $b_{\alpha, \beta} \in F_{0}$.

Démonstration. - Pour démontrer cette proposition (par récurrence) on a besoin :

- des formules suivantes :

$$
\begin{aligned}
& \frac{\partial y}{\partial \theta}=\varphi, \quad \frac{\partial y}{\partial t}=\theta \varphi \varphi^{\prime}, \quad \frac{\partial x}{\partial \theta}=0, \quad \frac{\partial x}{\partial t}=\varphi, \\
& \frac{\partial \theta}{\partial y}=\frac{1}{\varphi}, \quad \frac{\partial t}{\partial y}=0, \quad \frac{\partial \theta}{\partial x}=-\frac{\varphi^{\prime} \theta}{\varphi}, \quad \frac{\partial t}{\partial x}=\frac{1}{\varphi} ;
\end{aligned}
$$

- de la propriété : si $e$ appartient à $E_{J}$, alors $\varphi \partial_{x} e$ appartient à $E_{J+1}$;

- de la définition des éléments de $F_{0}$. 
Corollaire 2.18. - Soit

$$
L u=\sum_{|\alpha| \leq 2 m} \varphi^{-|\alpha|}\left(a_{\alpha}(0,0) \partial_{t, \theta}^{\alpha}+\sum_{|\beta| \leq|\alpha|} C_{\alpha, \beta} \partial_{t, \theta}^{\beta}\right) v
$$

$\operatorname{avec} C_{\alpha, \beta} \in F_{0}$.

Corollaire 2.19. - On a :

$$
\varphi^{2 m} L u(x, y)=L_{0}\left(\partial_{t}, \partial_{\theta}\right) v+P\left(t, \theta, \partial_{t}, \partial_{\theta}\right) v
$$

où $P=\sum_{|\gamma| \leq 2 m} C_{\gamma} \partial_{t, \theta}^{\gamma}$ avec $C_{\gamma} \in F_{0}$.

DÉFINITION 2.20.- Si $a \in \mathbb{R}$ et $n \in \mathbb{N}$,

$$
v \in H_{a}^{n}(\Omega) \Longleftrightarrow \varphi^{a} v \in H^{n}(\Omega) .
$$

THÉORÈME 2.21.- Soit

$$
v \in V_{a}^{n}(U) \Longleftrightarrow v=u \circ \mathcal{C}^{-1} \circ \mathcal{T}^{-1} \in H_{-n+a+1}^{n}(\Omega) .
$$

Ce théorème découle du corollaire 2.14 , de la proposition 2.17 et du résultat " réciproque ": pour tout multi-indice $\alpha$, on a

$$
\partial_{t, \theta}^{\alpha} v=\varphi^{|\alpha|}\left(\partial_{x, y}^{\alpha} \widetilde{u}+\sum_{|\beta| \leq|\alpha|} d_{\alpha, \beta} \partial_{x, y}^{\beta} \tilde{u}\right), \quad d_{\alpha, \beta} \in F_{0} .
$$

III. Propriétés sur les espaces

Proposition 2.22. - Si $u \in \stackrel{\circ}{H}^{m}(U)$, alors $u \in V_{m-1}^{m}(U)$.

Cette proposition se démontre assez facilement en utilisant l'inégalité de Schwarz, pour $u$ appartenant à $\mathcal{D}(U)$, à

$$
u(x, z)=\int_{\varphi_{1}(x)}^{z} \frac{\partial u}{\partial y}(x, y) \mathrm{d} y
$$

et en intégrant $|u(x, z)|^{2}$ par rapport à $z$ sur $\left[\varphi_{1}(x), \varphi_{2}(x)\right]$. 
Problème de Dirichlet pour un opérateur elliptique dans un domaine à point cuspide

Proposition 2.23. - Si $n \geq 3$, on a $V^{n}(U) \subset C^{n-2}(\bar{U})$.

Pour démontrer cette proposition on utilise le lemme suivant.

Lemme 2.24. - Si $a \in \mathbb{R}, n \in \mathbb{N}$, on a

$$
\varphi^{a} v \in W^{n, \infty}(\Omega) \quad \Longleftrightarrow \quad \forall|\alpha| \leq n, \varphi^{a} \partial_{t, \theta}^{\alpha} \in W^{0, \infty}(\Omega) .
$$

Ce lemme se montre facilement en utilisant que $\forall n \in \mathbb{N}$,

$$
\partial_{t}^{n} \varphi^{a}=\varphi^{a} e_{n}, \quad e_{n} \in W^{0, \infty}(\Omega) .
$$

Démonstration de la proposition. - Si $u \in V^{n}(U)$, alors

$$
\varphi^{-n+1} u \circ \mathcal{C}^{-1} \circ \mathcal{T}^{-1}=\varphi^{-n+1} v \in H^{n}(\Omega) .
$$

L'injection de Sobolev donne

$$
\varphi^{-n+1} v \in W^{n-2, \infty}(\Omega) \cap C^{n-2}(\bar{\Omega}) .
$$

D'après le lemme précédent on $\mathrm{a}, \forall \alpha$ tel que $|\alpha| \leq n-2$,

$$
\varphi^{-n+1} \partial^{\alpha} v \in W^{0, \infty}(\Omega) \cap C^{0}(\bar{\Omega}) .
$$

On utilise alors les propositions 2.17 puis 2.11 pour obtenir que $\forall \alpha$ tel que $|\alpha| \leq n-2$,

$$
\varphi^{|\alpha|-n+1} \partial_{x, y}^{\alpha} v \in W^{0, \infty}(U) \cap C^{0}(\bar{U} \backslash\{0\}) .
$$

Il suffit ensuite d'utiliser que $|\alpha|-n+1<0$, donc que $\varphi^{|\alpha|-n+1} \rightarrow+\infty$ quand $x \rightarrow 0, \forall \alpha$ tel que $|\alpha| \leq n-2$ pour obtenir le résultat.

Remarque 2.25. - On ne peut pas appliquer directement les injections de Sobolev sur $U$ car il existe $\varepsilon>0$ et $u \in H^{n}(U)$ avec $u \notin C^{n-1-\varepsilon}(\bar{U})$ : par exemple si $U$ est de la forme

$$
U \cap B(0, \varepsilon)=\left\{(x, y) \in B(0, \varepsilon) \mid 0<y<x^{p}\right\},
$$

il suffit de prendre $u=x^{n-1-y}$ avec $(1 / 2)(p-1)>\gamma>0$ car on a

$$
u \in H^{n}(U) \Longleftrightarrow p-1-2 \gamma>0
$$

et par contre, dès que $0<\varepsilon<\gamma$, on a $u \notin C^{n-1-\varepsilon}(\bar{U})$. 
Corollaire 2.26. - Si

$$
u \in \bigcap_{n \in \mathbb{N}} V^{n}(U)
$$

alors $u \in C_{\infty}^{\infty}(U)$.

Ce corollaire découle facilement de la proposition 2.23 .

Proposition 2.27. - Sous l'hypothèse $\left(H_{1}\right)$, si $n \geq 1$, si $f \in C^{\infty}(\bar{U})$ et si

$$
f_{n}=\sum_{k+\ell \leq[p n]-1} \frac{1}{k !} \frac{1}{\ell !} x^{k} y^{\ell}\left(\partial_{x}^{k} \partial_{y}^{\ell} f\right)(0,0),
$$

alors

$$
f-f_{n} \in V^{n}(U) \text {. }
$$

Cette proposition s'obtient en appliquant pour $\alpha$ tel que $|\alpha| \leq n$, la formule de Taylor en 0 à l'ordre $k=[p n]-[\alpha]$ à la fonction $\partial^{\alpha} f$ sur un voisinage de $U$ et en utilisant l'inégalité qui découle de l'hypothèse $\left(\mathrm{H}_{1}\right)$.

\section{Régularité dans les espaces à poids}

\subsection{Démonstration du théoréme 1.1}

D'aprés le corollaire 2.19 l'équation $(0.1)$ avec $u \in \stackrel{\circ}{H}^{m}(U)$ et $f \in V^{n}(U)$ se transforme en

$$
\left(L_{0}+p\right) v=g
$$

avec $g=\left(\varphi^{2 m} f\right) \circ(\mathcal{T} \circ \mathcal{C})^{-1}$. D'aprés les propositions 2.21 et 2.22 , $v \in H^{m}(\Omega)$ et $g \in H_{-n+1-2 m}^{n}(\Omega)$.

Soit $\zeta_{0}$ une fonction $C^{\infty}$ sur $\mathbb{R}$ telle que

$$
\zeta_{0}(t)= \begin{cases}1 & \text { si } t \leq-1 \\ 0 & \text { si } t \geq 0\end{cases}
$$

On posera dans la suite, pour tout $T$ réel,

$$
\begin{aligned}
\zeta_{T}(t) & =\zeta_{0}(t+T) \\
& -162-
\end{aligned}
$$


Problème de Dirichlet pour un opérateur elliptique dans un domaine à point cuspide

\section{Première étape}

Soit $B=\{(t, \theta) \mid t \in \mathbb{R}, \theta \in] 0,1[\}$.

Proposition 3.1.- Sous l'hypothèse $(\mathcal{L})$ pour $L, L_{0}$ est un isomorphisme de $H^{k+2 m} \cap \stackrel{\circ}{H}^{m}(B)$ sur $H^{k}(B)$ pour tout $k$ de $\mathbb{Z}$ tel que $k \geq-m$.

Pour démontrer cette proposition on utilise le lemme classique suivant (cf. [8] par exemple).

Lemme 3.2.- Il existe $K$ et $\lambda_{1}$ tels que pour tout $\lambda \in \mathbb{R}$ tel que $|\lambda| \geq \lambda_{1}$ on ait :

$$
\begin{aligned}
& \forall u \in H^{k+2 m} \cap \stackrel{\circ}{H}^{m}(] 0,1[), \\
& \sum_{0 \leq \ell \leq k+2 m}\left(1+\lambda^{2}\right)^{k+2 m-\ell}\left\|d^{\ell} u\right\|_{L^{2}(] 0,1[)}^{2} \leq \\
& \leq K \sum_{0 \leq \ell \leq k}\left(1+\lambda^{2}\right)^{k+\ell}\left\|L_{0}(\lambda, \mathrm{d}) u\right\|_{L^{2}(] 0,1[)}^{2} .
\end{aligned}
$$

Grâce à cette estimation a priori on obtient que pour $\lambda$ grand, $L_{0}(\lambda, \mathrm{d})$ est à image fermée d'où :

$$
\left[\operatorname{Im} L_{0}(\lambda, \mathrm{d})\right]^{\perp}=\operatorname{Ker}\left[L_{0}^{*}(\lambda, \mathrm{d})\right]
$$

en notant $L_{0}^{*}(\lambda, \mathrm{d})$ l'adjoint formel de $L_{0}(\lambda, \mathrm{d})$. Mais $L_{0}^{*}(\lambda, \mathrm{d})$ étant aussi proprement elliptique à coefficients constants vérifie l'inégalité a priori. $L_{0}^{*}(\lambda, \mathrm{d})$ est donc aussi injectif pour $\lambda$ grand. Ainsi $L_{0}(\lambda, \mathrm{d})$ est surjectif donc bijectif pour $\lambda$ grand. Il est donc à indice et d'indice nul. Soit $\lambda$ réel tel que $|\lambda| \leq \lambda_{1}$. Si on prend $\lambda^{\prime}$ tel que $\left|\lambda^{\prime}\right| \geq 1, L_{0}(\lambda, \mathrm{d})=$ $L_{0}\left(\lambda^{\prime}, \mathrm{d}\right)+P\left(\lambda, \lambda^{\prime}, \mathrm{d}\right)$ où $P\left(\lambda, \lambda^{\prime}, \mathrm{d}\right)$ est un opérateur d'ordre $2 m-1$ donc compact de $H^{k+2 m} \cap \stackrel{\circ}{H}^{m}(] 0,1[)$, dans $H^{k}(] 0,1[) . L_{0}(\lambda, \mathrm{d})$ est donc d'indice nul. Comme il est injectif, il est par conséquent bijectif.

Remarque 3.3.- On a ainsi obtenu que pour tout réel $\lambda, L_{0}(\lambda, \mathrm{d})$ est bijectif sur $\stackrel{\circ}{H}^{m}(] 0,1$ [). Pour obtenir la proposition 3.1 on utilise alors que $\mathcal{F}$ défini par :

$$
\mathcal{F} v(\lambda, \theta)=\int_{-\infty}^{+\infty} e^{-i t \lambda} v(t, \theta) \mathrm{d} t
$$


est un isomorphisme de $H^{k}(B) \operatorname{sur} V^{k}(B)$ avec

$$
\begin{aligned}
& V^{k}(B)= \\
& =\left\{v \in L^{2}(B) \mid\left(1+\lambda^{2}\right)^{j / 2} \partial_{\theta}^{\ell} v(\lambda, \theta) \in L^{2}(B), \forall j, \ell \in \mathbb{N}, j+\ell \leq k\right\}
\end{aligned}
$$

(l'injection découle de l'injection de $L_{0}(\lambda, \mathrm{d})$ pour tout $\lambda$ de $\mathbb{R}$; la surjection est une conséquence de l'estimation a priori que l'on intègre sur $\mathbb{R}$, par rapport à $\lambda$ ).

\section{Deuxième étape}

Montrons que $v$ appartient à $H^{n+2 m}(\Omega)$.

LEMME 3.4. - Si $Q=\sum_{0 \leq|\gamma| \leq 2 m} b_{\gamma} \partial_{t, \theta}^{\gamma}$ où $b_{\gamma} \in F_{0}$, alors la norme de l'opérateur $\zeta_{\delta} Q$ dans $\mathcal{L}\left(H^{k+2 m}(B), H^{k}(B)\right)$ tend vers 0 lorsque $\delta$ tend vers $+\infty, \forall k \geq-m$.

Démonstration. - Ce lemme est une conséquence du résultat suivant qui se démontre par récurrence sur $\alpha$ :

$$
\forall \alpha, \quad \partial_{t, \theta}^{\alpha}\left(\zeta_{\delta} Q \nu\right)=\sum_{q \leq|\alpha|} \sum_{|\gamma| \leq|\alpha|+2 m} b_{q, \gamma} \frac{\mathrm{d}^{\mathrm{q}}}{\mathrm{d} t^{q}}\left(\zeta_{\delta}\right) \partial_{t, \theta}^{\gamma} v
$$

où $b_{q, \gamma} \in F_{0}$.

En effet, d'après la remarque $2.16, e$ tend vers 0 quand $x$ tend vers 0 si $e$ appartient à $F_{0}$, d'où

$$
\forall k \in \mathbb{N}, \quad \sum_{|q| \leq k} \sum_{|\gamma| \leq k+2 m}\left|\frac{\partial^{q} \zeta_{\delta}}{\partial t^{q}}\right|\left|b_{q, \gamma}\right|
$$

tend vers 0 quand $\delta$ tend vers $+\infty$.

Par conséquent si $\delta$ est suffisamment grand, $L_{0}+\zeta_{\delta} P$ est un isomorphisme de $H^{k+2 m} \cap \stackrel{\circ}{H}^{m}(B)$ sur $H^{k}(B)$ pour tout $k$ de $\mathbb{Z}, k \geq-m$ ( $P$ est à coefficients dans $F_{0}$ ). On a, pour tout $T$ réel,

$$
L\left(\zeta_{T} v\right)=\zeta_{T} L v+\sum_{0<|\alpha| \leq 2 m} c_{\alpha} \partial^{\alpha} \zeta_{T}
$$

où les $c_{\alpha}$ sont des combinaisons des coefficients de $L$ et de dérivées d'ordre inférieur à $2 m$ de $v$. Ainsi

$$
L\left(\zeta_{T} v\right)=\zeta_{T} g+\sum_{0<|\alpha| \leq 2 m} c_{\alpha} \partial^{\alpha} \zeta_{T}
$$


Problème de Dirichlet pour un opérateur elliptique dans un domaine à point cuspide

Posons

$$
\zeta_{T} g+\sum_{0<|\alpha| \leq 2 m} c_{\alpha} \partial^{\alpha} \zeta_{T}=h
$$

Si l'on suppose que $v$ appartient à $H^{k}(B)$ avec $m \leq k<n+2 m$, alors $h$ appartient à $H^{k+1-2 m}(B)$. Si $T$ est assez grand

$$
\left(1-\zeta_{\delta}\right) P\left(\zeta_{T} v\right)=0
$$

d'où

$$
\left(L_{0}+\zeta_{\delta} P\right)\left(\zeta_{T} v\right)=h-\left(1-\zeta_{\delta}\right) P\left(\zeta_{T} v\right)=h
$$

Ainsi, d'après le lemme 3.4 si $\delta$ est assez grand, $\zeta_{T} v$ appartient à $H^{k+1}(B)$. Finalement, comme $\left(1-\zeta_{T}\right) v$ appartient à $H^{n+2 m}(B)$ pour tout $T$ réel, $v$ appartiendra à $H^{k+1}(\Omega)$. Comme $v$ appartient à $H^{m}(\Omega)$, $v$ appartient à $H^{n+2 m}(\Omega)$.

\section{Troisième étape}

Montrons que $v$ appartient à $H_{-n-2 m+1}^{n+2 m}(\Omega)$.

Posons $w=\varphi^{-n-2 m+1} v$. La fonction $w$ appartient à $H_{+n+2 m-1}^{n+2 m}(\Omega)$. Comme $(\partial / \partial \theta)\left(\varphi^{n+2 m-1}\right)=0$ et que

$$
\forall k \in \mathbb{N}, \forall b \in \mathbb{R}, \quad \frac{\partial}{\partial t^{k}}\left(\varphi^{b}\right)=\varphi^{b} e_{k}, \quad e_{k} \in E_{k},
$$

on a

$$
\left(L_{0}+P\right)\left(\varphi^{n+2 m-1} w\right)=\varphi^{n+2 m-1}\left(L_{0}+R\right) w=g
$$

où les coefficients de $R$ appartiennent à $F_{0}$. Ainsi

$$
\left(L_{0}+\zeta_{T} R\right) w=\varphi^{-n-2 m+1} g-\left(1-\zeta_{T}\right) R w .
$$

Mais

$$
\varphi^{-n+2 m+1} g-\left(1-\zeta_{T}\right) R w \in H^{n}(\Omega)
$$

d'où en utilisant le lemme 3.4 on obtient que pour $T$ assez grand, $w$ appartient à $H^{n+2 m}(\Omega)$. Ce qui donne $v$ appartient à $H_{-n-2 m+1}^{n+2 m}(\Omega)$, c'està-dire, d'après le théorème 2.21, $u$ appartient à $V^{n+2 m}(U)$. Ce qui termine la démonstration du théorème 1.1 . 


\section{Jean-Luc Steux}

Preuve du corollaire 1.2. - Si $u$ appartient à $\stackrel{\circ}{H}^{m}(U)$ est tel que $L u=\lambda u$ sur $U$, alors $\lambda u$ appartient à $L^{2}(U)$; d'où en appliquant le théorème 1.1, nous avons

$$
u \in V^{2 m}(U)
$$

donc

$$
\lambda u \in V^{2 m}(U)
$$

En répétant le raisonnement nous obtenons que

$$
u \in \bigcap_{n \in \mathbb{N}} V^{n}(U)
$$

Le corollaire 1.2 se déduit alors du corollaire 2.26 . Le théorème 1.3 est une conséquence immédiate du théorème 1.1 et de la proposition 2.27 : en effet si $f$ appartient à $C^{\infty}(\bar{U}), f$ appartient à $\bigcap_{n \in \mathbb{N}} V^{n}(U)$.

\section{Résolution polynomiale approchée}

Dans toute la suite, nous ferons l'hypothèse $\left(\mathrm{H}_{2}\right)$. Nous introduirons les espaces suivants qui engloberont les singularités et les polynômes.

DÉfINITION 4.1. - Soient $i, k, \ell, a \in \mathbb{N}, j \in \mathbb{Z}$. On appelle $\mathcal{S}^{(i, j, k, \ell, a)}$ (resp. $\widetilde{\mathcal{S}}^{(i, j, k, \ell, a)}$ ) l'espace des expressions de la forme

$$
x^{i} \varphi^{j} \varphi_{1}^{k} e\left(y-\varphi_{1}\right)^{a} \quad\left(r e s p . X^{i} \varphi^{j} \varphi_{1}^{k} e Y^{a}\right)
$$

où $e \in E_{\ell}$ et où la puissance de $\varphi$ dans chacune des ces expressions est positive ( $\varphi$ apparaît dans $\varphi^{j}$ et dans e).

Soient $s \in \mathbb{R}, \lambda \in \mathbb{N}$, on appelle $\mathcal{S}^{s, \lambda}$ (resp. $\widetilde{\mathcal{S}}^{s, \lambda}$ ) les combinaisons linéaires finies d'éléments de $\mathcal{S}^{(i, j, k, \ell, a)}$ (resp. $\left.\widetilde{\mathcal{S}}^{(i, j, k, \ell, a)}\right)$ telles que

$$
\frac{i}{p}+j+\frac{k}{p^{\prime}}+A(\ell)+a \geq s, \quad \ell \leq \lambda .
$$

Soit $s \in \mathbb{R}$, on appelle $\mathcal{S}^{s}$ (resp. $\widetilde{S}^{s}$ ) les combinaisons linéaires finies d'éléments de $\mathcal{S}^{(i, j, k, \ell, a)}$ (resp. $\left.\widetilde{\mathcal{S}}^{(i, j, k, \ell, a)}\right)$ telles que

$$
\frac{i}{p}+j+\frac{k}{p^{\prime}}+A(\ell)+a \geq s .
$$


Problème de Dirichlet pour un opérateur elliptique dans un domaine à point cuspide

LEMME $4.2 .-\quad S i u_{1} \in \widetilde{\mathcal{S}}^{s_{1}, \lambda_{1}}$ et $u_{2} \in \widetilde{\mathcal{S}}^{s_{2}, \lambda_{2}}$, alors $u_{1} \times u_{2} \in$ $\widetilde{\mathcal{S}}^{s_{1}}+s_{2}, \lambda_{1}+\lambda_{2}$.

Ce lemme provient de la décroissance des $\delta(N), N \in \mathbb{N}^{*}$ (définis dans l'hypothèse $\left.\left(\mathrm{H}_{2}\right)\right)$ qui entraîne que $A\left(\ell_{1}\right)+A\left(\ell_{2}\right) \geq A\left(\ell_{1}+\ell_{2}\right)$ si $\ell_{1}, \ell_{2} \in \mathbb{N}$.

Remarque 4.3

- Dans le cas de l'hypothèse $\left(\mathrm{H}_{4}\right)$ on a $\mathcal{S}^{(i, j, k, \ell, a)} \subset C^{\infty}(\bar{U})$ d'où $\mathcal{S}^{s} \subset C^{\infty}(\bar{U})$.

- Nous verrons que lorsque $s$ augmente, la régularité des éléments de $\mathcal{S}^{s}$ augmente.

LEMmE 4.4. - Si $\widetilde{S} \in \mathcal{C}_{d, \lambda}$ avec $d \in \mathbb{N}$, alors $\widetilde{S} \in \widetilde{\mathcal{S}}^{\delta(1)-d, d+\lambda)}$.

Démonstration. - Si $\widetilde{S}=\varphi_{1}^{\left(n_{1}+1\right)} \cdots \varphi_{1}^{\left(n_{k}+1\right)} \in \mathcal{C}_{d, \lambda}$, alors

$$
\varphi^{d} \widetilde{S}=\varphi^{d-\left(n_{1}+\cdots+n_{k}\right)} e
$$

où $e \in E_{n_{1}+1+\cdots+n_{k}+1}$ avec $k \geq 1, n_{1}, \ldots, n_{k} \geq 0$,

$$
n_{1}+\cdots+n_{k} \leq d, \quad k \leq \lambda
$$

Les puissances de $\varphi$ dans $\widetilde{S}$ étant nulles nous avons le résultat.

Proposition 4.5.- Si $S \in \mathcal{S}^{s, \lambda}$, alors

$$
\partial_{x}^{\alpha} \partial_{y}^{\beta} S \in \mathcal{S}^{s-\alpha+\alpha \delta(\lambda+\alpha)-\beta, \alpha+\lambda}
$$

Démonstration. - Pour obtenir cette proposition on démontre d'abord par récurrence sur $d$ que si $S \in \mathcal{S}^{(i, j, k, \ell, a)}$ et $d \in \mathbb{N}$, alors $\partial_{x}^{d} S$ appartient à la somme des espaces

$$
\mathcal{S}^{\left(i^{(\alpha)}, j^{(\alpha)}, k^{(\alpha)}, \ell^{(\alpha)}, a^{(\alpha)}\right)}, \quad \text { où } \alpha=\left(\alpha_{1}, \alpha_{2}, \alpha_{3}, \alpha_{4}\right) \in \mathbb{N}^{4},|\alpha|=d,
$$

et

$$
\begin{aligned}
& i^{(\alpha)}=i-\alpha_{1}, \quad j^{(\alpha)}=j-\alpha_{2}, \quad k^{(\alpha)}=k-\alpha_{3}, \\
& \ell^{(\alpha)}=\ell+\alpha_{2}+\alpha_{3}+\alpha_{4}, \quad a^{(\alpha)}=a-\alpha_{4}, \quad i^{(\alpha)}, k^{(\alpha)} \geq 0 .
\end{aligned}
$$




\section{Jean-Luc Steux}

Ainsi si $S \in \mathcal{S}^{s, \lambda}, \partial_{x}^{\alpha} \partial_{y}^{\beta} S$ appartient à la somme des espaces

$$
\mathcal{S}^{\left(i^{(\alpha)}, j^{(\alpha)}, k^{(\alpha)}, \ell^{(\alpha)}, a^{(\alpha)}-\beta\right)}
$$

avec $\alpha_{1}, \alpha_{2}, \alpha_{3}, \alpha_{4} \in \mathbb{N}, \alpha_{1}+\alpha_{2}+\alpha_{3}+\alpha_{4}=\alpha$, et

$$
\frac{i}{p}+j+\frac{k}{p}+A(\ell)+a \geq s, \quad \ell \leq \lambda .
$$

On a ainsi l'égalité

$$
\begin{aligned}
& \frac{i^{(\alpha)}}{p}+j^{(\alpha)}+\frac{k^{(\alpha)}}{p^{\prime}}+A\left(\ell^{(\alpha)}\right)+a^{(\alpha)}-\beta= \\
& \quad=\frac{i}{p}+j+\frac{k}{p^{\prime}}+A\left(\ell+\alpha_{2}+\alpha_{3}+\alpha_{4}\right)+a-\frac{\alpha_{1}}{p}-\alpha_{2}-\frac{\alpha_{3}}{p^{\prime}}-\frac{\alpha_{4}}{p}-\beta .
\end{aligned}
$$

Puisque $\alpha_{2}+\alpha_{3}+\alpha_{4} \leq \alpha$ et que la suite $(\delta(N))_{N \geq 1}$ est décroissante, on a

$$
\begin{aligned}
A\left(\ell+\alpha_{2}+\alpha_{3}+\alpha_{4}\right)-A(\ell) & \geq\left(\alpha_{2}+\alpha_{3}+\alpha_{4}\right) \delta\left(\ell+\alpha_{2}+\alpha_{3}+\alpha_{4}\right) \\
& \geq\left(\alpha_{2}+\alpha_{3}+\alpha_{4}\right) \delta(\ell+\alpha) .
\end{aligned}
$$

d'où

$$
\begin{aligned}
(4.3) & \geq s-\beta+\left(\alpha_{2}+\alpha_{3}+\alpha_{4}\right) \delta(\ell+\alpha)-\frac{\alpha_{1}}{p}-\alpha_{2}-\frac{\alpha_{3}}{p^{\prime}}-\frac{\alpha_{4}}{p} \\
& \geq s-\alpha+\alpha_{1}\left(1-\frac{1}{p}\right)+\left(\alpha_{2}+\alpha_{3}+\alpha_{4}\right) \delta(\ell+\alpha)-\beta .
\end{aligned}
$$

D'autre part, $\delta(\ell+\alpha) \leq 1-1 / p$ entraîne

$$
\text { (4.3) } \geq s-\alpha+\alpha \delta(\ell+\alpha)-\beta .
$$

Comme $\ell \leq \lambda$, on obtient le résultat désiré.

On déduit de la proposition précédente et de la proposition 2.1 le résultat suivant.

Proposition 4.6. - Si $S \in \mathcal{S}^{s}$ et si $\left(\varphi_{1}, \varphi_{2}\right)$ vérifie l'hypothèse $\left(H_{2}\right)$, alors

$$
\partial_{x}^{\alpha} \partial_{y}^{\beta} S \in \mathcal{S}^{s-\beta-\alpha} .
$$

Si $\left(\varphi_{1}, \varphi_{2}\right)$ vérifie l'hypothèse $\left(H_{3}\right)$, alors

$$
\partial_{x}^{\alpha} \partial_{y}^{\beta} S \in \mathcal{S}^{s-\beta-\alpha\left(p-p_{2}+1\right) / p} .
$$


Problème de Dirichlet pour un opérateur elliptique dans un domaine à point cuspide

Proposition 4.7.- Soit $n \in \mathbb{N}$. Si $s \geq n-1 / 2$, alors, $\forall \lambda \in \mathbb{N}$, $\widetilde{\mathcal{S}}^{s, \lambda} \subset V^{n}(G)$.

Pour démontrer cette proposition on utilise la définition de $\widetilde{\mathcal{S}}^{s, \lambda}$, les inégalités $X \leq \varphi^{1 / p},\left|\varphi_{1}(X)\right| \leq C \varphi(X)^{1 / p^{\prime}}$ et ainsi que la proposition 4.6.

La proposition 4.7 justifie la définition suivante.

DÉfinition 4.8. - Si $s \in \mathbb{R}$ et $\lambda, n \in \mathbb{N}$ avec $n>s+1 / 2$, on appelle $T_{s, \lambda, n}$ la somme des espaces $\widetilde{\mathcal{S}}^{s, \lambda}$ et $V^{n}(G)$. Si $n \leq s+1 / 2, T_{s, \lambda, n}$ désignera $V^{n}(G)$.

Remarque 4.9. - Le produit d'un élément de $\mathcal{S}^{s, \lambda}$ et d'un élément de $T_{s^{\prime}, \lambda^{\prime}, n}$ est dans $T_{s+s^{\prime}, \lambda+\lambda^{\prime}, n}$.

LeMme 4.10.- Si $f \in C^{\infty}(\bar{U})$ et $b \in \mathbb{N}$

$$
f-\sum_{|\alpha| \leq b} \frac{1}{\alpha !} x^{\alpha_{1}} y^{\alpha_{2}} f^{(\alpha)}(0) \in T_{(b+1) / p, 0, n} \quad \forall n \in \mathbb{N} .
$$

Démonstration. - D'après la proposition 2.6 , si $n \geq 1$ et si $f \in C^{\infty}(\bar{U})$

$$
f-\sum_{|\alpha| \leq[p n]-1} \frac{1}{\alpha !} x^{\alpha_{1}} x^{\alpha_{2}} f^{(\alpha)}(0) \in V^{n}(U) \text {. }
$$

Or

$$
x^{\alpha_{1}} y^{\alpha_{2}}=\sum_{0 \leq k \leq \alpha_{2}} C_{\alpha_{2}}^{k} X^{\alpha_{1}} Y^{\alpha_{2}-k} \varphi_{1}^{k}
$$

avec

$$
X^{\alpha_{1}} Y^{\alpha_{2}-k} \varphi_{1}^{k} \in \widetilde{\mathcal{S}}^{\left(\alpha_{1}, 0, k, 0, \alpha_{2}-k\right)}
$$

Comme $p^{\prime} \leq p$ on a, lorsque $b<[p n]$,

$$
\sum_{b+1 \leq|\alpha| \leq[p n]-1} \frac{1}{\alpha !} x^{\alpha_{1}} x^{\alpha_{2}} f^{(n)}(0) \in \widetilde{\mathcal{S}}^{(b+1) / p, 0} . \square
$$


Proposition 4.11. - Lu $=\tilde{L} \tilde{u}$ avec

$$
\widetilde{L}=L_{0}\left(\partial_{X}, \partial_{Y}\right)+\sum_{|\alpha| \leq 2 m} c_{\alpha} \partial_{X, Y}^{\alpha}
$$

où $c_{\alpha} \in T_{q+|\alpha|-2 m, 4 m, n}, \forall n \in \mathbb{N}$, avec $q=\inf (\delta(1), 1 / p)$.

Cette proposition découle des lemmes 4.4, 4.10 et du corollaire 2.12 .

DÉfinition 4.12.- On appelle $\widetilde{\mathcal{S}}_{0}^{s}\left(\right.$ resp. $\widetilde{\mathcal{S}}_{0}^{s, \lambda}$ ) les éléments $\widetilde{S}$ de $\widetilde{\mathcal{S}}^{s}$ (resp. $\tilde{\mathcal{S}}^{s, \lambda}$ ) tels que

$$
\chi(\widetilde{\mathcal{S}} \circ \mathcal{C}) \in \stackrel{\circ}{H}^{m}(U)
$$

où $\chi$ est la fonction définie dans le lemme 1.5.

Nous commençons maintenant la résolution avec second membre polynomial ou singulier.

LEMME 4.13. - $\forall \widetilde{S} \in \widetilde{\mathcal{S}}^{s, \lambda}$, il existe $\widetilde{B} \in \widetilde{\mathcal{S}}_{0}^{s+2 m, \lambda}$ tel que $\partial_{Y}^{2 m} \widetilde{B}=\widetilde{S}$.

Démonstration. - Tout générateur de $\widetilde{\mathcal{S}}^{s, \lambda}$ est de la forme $\widetilde{S}=f(X) Y^{a}$ et, par intégration en $Y$, on contruit une solution $\widetilde{B}$ qui lui correspond, elle a la forme

$$
f(X)\left(c_{a} Y^{a+2 m}-\sum_{0 \leq j \leq m-1} c_{a, j} \varphi^{a+m-j} Y^{m+j}\right)
$$

dont on vérifie immédiatement qu'elle appartient à $\widetilde{\mathcal{S}}_{0}^{s+2 m, \lambda}$.

En utilisant ce lemme, les propositions $4.5,4.11$ et la remarque 4.9 , on obtient le résultat suivant.

Proposition 4.14. - Soit $\widetilde{S} \in \widetilde{\mathcal{S}}^{s, \lambda}$. Alors il existe

$$
\widetilde{B}_{1} \in \widetilde{\mathcal{S}}_{0}^{s+2 m, \lambda}, \quad R_{1} \in T_{s+q^{\prime}, \lambda+6 m, n}
$$

avec $q^{\prime}=\inf (\delta(\lambda+2 m), 1 / p)$ vérifiant

$$
\widetilde{L} \widetilde{B}_{1}=\widetilde{S}+R_{1} .
$$

Une application itérée de la proposition 4.14 donne la proposition qui suit. 
Problème de Dirichlet pour un opérateur elliptique dans un domaine à point cuspide

Proposition 4.15.- Soit $\widetilde{S} \in \widetilde{\mathcal{S}}^{s, \lambda}$. Alors $\forall k \in \mathbb{N}^{*}$ il existe $\widetilde{B}_{1}, \widetilde{B}_{2}, \ldots, \widetilde{B}_{k}, j_{1}, j_{2}, \ldots, j_{k}$ tels que

$$
\begin{gathered}
\widetilde{B}_{1} \in \widetilde{\mathcal{S}}_{0}^{s+2 m, \lambda}, \ldots, \widetilde{B}_{k} \in \widetilde{\mathcal{S}}_{0}^{s+2 m+j_{k-1}, \lambda+(k-1) 6 m} \\
j_{0}=0, \quad j_{i}=j_{i-1}+\inf \left(\delta(\lambda+(i-1) 6 m+2 m), \frac{1}{p}\right), \quad 1 \leq i \leq k \\
R_{k} \in T_{s+j_{k}, \lambda+6 m k, n} \\
\widetilde{L}\left(\widetilde{B}_{1}+\widetilde{B}_{2}+\cdots+\widetilde{B}_{k}\right)=\widetilde{S}+R_{k} .
\end{gathered}
$$

Remarque. - Comme on a fait l'hypothèse $\sum_{1 \leq N \leq+\infty} \delta(N)=+\infty$, la suite $\left(j_{k}\right)_{k \geq 1}$ tend vers $+\infty$.

Démonstration des théorèmes 1.4 et 1.6

Soit

$$
f_{n}=\sum_{|\alpha| \leq[p n]-1} \frac{1}{\alpha !} x^{\alpha_{1}} y^{\alpha_{2}}\left(\partial^{\alpha} f\right)(0,0)
$$

D'après la proposition 2.27, $f-f_{n} \in V^{n}(U)$,

$$
x^{\alpha_{1}} y^{\alpha_{2}}=X^{\alpha_{1}}\left(Y+\varphi_{1}\right)^{\alpha_{2}} \in \widetilde{\mathcal{S}}^{\alpha_{1} / p+\alpha_{2} / p^{\prime}}
$$

d'où en appliquant la proposition précédente

$$
\widetilde{S}=\left(x^{\alpha_{1}} y^{\alpha_{2}}\right) \circ \mathcal{C}, \quad s=\frac{\alpha_{1}}{p}+\frac{\alpha_{2}}{p^{\prime}}
$$

et en prenant $K$ suffisamment grand pour que

$$
R_{k} \in V^{n}(U)
$$

on obtient, en posant $\sigma_{\alpha, n}=\widetilde{B}_{1}+\widetilde{B}_{2}+\cdots+\widetilde{B}_{k}$ que

$$
L\left(S_{n}\right)=f_{n}+R_{n}^{\prime}, \quad R_{n}^{\prime} \in V^{n}(U) .
$$

Comme

$$
f-f_{n} \in V^{n}(U)
$$

d'après le théorème 1.1 ,

$$
u-S_{n} \in V^{n+2 m}(U)
$$


d'où le théorème 1.6. D'après la proposition 4.15, $\sigma_{\alpha, n}$ appartient à $\mathcal{S}^{\alpha_{1} / p+\alpha_{2} / p^{\prime}+2 m}$. Dans le cas de l'hypothèse $\left(\mathrm{H}_{4}\right)$ (remarque 4.3), $\sigma_{\alpha, n}$ appartient à $C^{\infty}(\bar{U})$, d'où, d'après la proposition 2.23 ,

$$
\forall n \in \mathbb{N}, \quad u \in C^{n+2 m-2}(\bar{U}) .
$$

On obtient ainsi le théorème 1.4.

En étudiant la régularité des $S_{n}$ lorsque l'hypothèse $\left(\mathrm{H}_{4}\right)$ n'est pas vérifiée on va obtenir les théorèmes 1.7 et 1.9 .

Proposition 4.16. - Soit $\widetilde{S}$ dans $\widetilde{\mathcal{S}}^{s}$. Alors si $\left(\varphi_{1}, \varphi_{2}\right)$ vérifie l'hypothèse $\left(H_{2}\right)$,

$$
\widetilde{S} \circ \mathcal{C} \in \begin{cases}C^{[s]}(\bar{U}) & \text { si } s \text { est non entier } \\ W^{s, \infty}(U) & \text { si } s \text { est entier. }\end{cases}
$$

Démonstration. - Soit $S=x^{i} \varphi^{j} \varphi_{1}^{k} e\left(y-\varphi_{1}\right)^{a}$ appartenant à $\mathcal{S}^{0}$. En utilisant que si $x$ est assez petit on a

$$
x^{p} \leq C_{1} \varphi, \quad\left|\varphi_{1}\right|^{k} \leq C_{2} \varphi^{k / p^{\prime}}, \quad e \leq C_{3} \varphi^{A(\ell)}, \quad\left|y-\varphi_{1}\right| \leq \varphi ;
$$

on obtient que $|S|$ est majoré pour $x$ petit par

$$
C_{4} \varphi^{i / p+j+k / p^{\prime}+A(\ell)+a} .
$$

Si $i / p+j+k / p^{\prime}+A(\ell)=0$, alors $S$ appartient à $W^{0, \infty}(U)$.

Si $i / p+j+k / p^{\prime}+A(\ell)>0$, alors $S$ appartient à $C^{\infty}(\bar{U})$.

On utilise alors la proposition 4.6 pour conclure.

Proposition 4.17. - Si $\widetilde{S} \in \widehat{\mathcal{S}}^{s}$ alors, si $\left(\varphi_{1}, \varphi_{2}\right)$ vérifie $\left(H_{3}\right)$

$$
\widetilde{S} \circ \mathcal{C} \in C^{a s, s}(\bar{U})
$$

où $a=p /\left(p-p_{2}+1\right)$.

Pour démontrer cette proposition, on utilise le lemme suivant.

Lemme 4.18. - Sous l'hypothèse $\left(H_{3}\right)$, si $\left.\sigma \in\right] 0,1[, n \in \mathbb{N}$ et sig est une fonction continue sur $\bar{U} \cap \mathbb{R}$, alors

$$
\begin{aligned}
g(x) x^{p(\sigma-n)}( & \left.y-\varphi_{1}(x)\right)^{n} \in C^{0, \sigma}(\bar{U}) . \\
- & 172-
\end{aligned}
$$


Problème de Dirichlet pour un opérateur elliptique dans un domaine à point cuspide

Si de plus $x g^{\prime}$ est bornée sur $\bar{U} \cap \mathbb{R}$, on a

$$
g(x) x^{\sigma-n p}\left(y-\varphi_{1}(x)\right)^{n} \in C^{\sigma, 0}(\bar{U})
$$

\section{Démonstration}

- Soient $x, y_{1}, y_{2}, y_{1} \neq y_{2}$ avec $\left(x, y_{1}\right),\left(x, y_{2}\right) \in \bar{U}$. On applique le théorème de Rolle à la fonction

$$
\begin{aligned}
\Phi_{1}(y)=( & \left.y-\varphi_{1}(x)\right)^{n}-\left(y_{1}-\varphi_{1}(x)\right)^{n}+ \\
& -\left(\left(y_{2}-\varphi_{1}(x)\right)^{n}-\left(y_{1}-\varphi_{1}(x)\right)^{n}\right)\left(y_{2}-y_{1}\right)^{-\sigma}\left(y-y_{1}\right)^{\sigma}
\end{aligned}
$$

pour obtenir (4.4), $\Phi_{1}\left(y_{1}-\varphi_{1}(x)\right)=\Phi_{2}\left(y_{2}-\varphi_{2}(x)\right)$.

- Si $\left(x_{1}, y\right),\left(x_{2}, y\right) \in \bar{U}$ avec $x_{1}<x_{2}$ (par exemple) on pose

$$
\Phi_{2}(x)=f(x, y)-f\left(x_{1}, y\right)-\left(f\left(x_{2}, y\right)-f\left(x_{1}, y\right)\right)\left(x_{2}-x_{1}\right)^{-\sigma}\left(x-x_{1}\right)^{\sigma}
$$

avec

$$
f(x, y)=g(x) x^{\sigma-n p}\left(y-\varphi_{1}(x)\right)^{n}
$$

Pour démontrer le lemme, comme $\Phi_{2}\left(x_{1}\right)=\Phi_{2}\left(x_{2}\right)=0$, on peut utiliser le théorème de Rolle et on obtient $c$ appartenant à ] $x_{1}, x_{2}$ [ tel que $\Phi_{2}^{\prime}(c)=0$. Pour obtenir (4.5), il suffit ensuite d'utiliser pour $\sigma \in] 0,1[$, on a

$$
\begin{gathered}
c^{\sigma-1}\left(c-x_{1}\right)^{\sigma-1} \leq 1 \\
c^{-n p}\left|y-\varphi_{1}(x)\right|^{n}, \quad c^{-n p+p}\left|y-\varphi_{1}(c)\right|^{n-1} \quad \text { bornés si } n \geq 1 \square .
\end{gathered}
$$

\section{Démonstration de la proposition 4.17}

Soient $\sigma, \tau \in[0,1[$ tels que

$$
\frac{p-p_{2}+1}{p} \sigma+\tau \leq s
$$

et soit $S_{1}=x^{i} \varphi^{j} \varphi_{1}^{k} e\left(y-\varphi_{1}\right)^{a}$ appartenant à $\mathcal{S}^{s}$.

- Dans le cas où $p_{1}=p_{2}, S_{1}$ est une combinaison linéaire de fonctions de la forme

$$
x^{\left(p-p_{2}+1\right) \sigma+p \tau-p b}\left(y-y_{1}\right)^{a} x^{b} .
$$


- Dans le cas où $p_{1}>p_{2}, S_{1}$ est une combinaison linéaire de fonctions de la forme

$$
x^{\sigma+p \tau-p b}\left(y-y_{1}\right)^{a} x^{b} .
$$

- Dans les deux cas, on peut conclure à l'aide du lemme précédent que $S_{1}$ appartient à $C^{\sigma, 0}(\bar{U}) \cap C^{0, \tau}(\bar{U})$. Si $\widetilde{S} \in \widetilde{\mathcal{S}}^{s}$, alors d'après la proposition 4.6, $\forall \alpha, \beta \in \mathbb{N}, \forall \sigma, \tau \in] 0,1[$ tels que

$$
\begin{gathered}
(\alpha+\sigma) \frac{p-p_{2}+1}{p}+\beta+\tau \leq s, \\
\partial_{x}^{\alpha} \partial_{y}^{\beta}(\tilde{S} \circ \mathcal{C}) \in S^{s-\beta-\alpha\left(p-p_{2}+1\right) / p}
\end{gathered}
$$

et comme

$$
\begin{gathered}
s-\beta-\alpha\left(p-p_{2}+1\right) p \geq \frac{p-p_{2}+1}{p} \sigma+\tau, \\
\partial_{x}^{\alpha} \partial_{y}^{\beta}(\widetilde{S} \circ \mathcal{C}) \in C^{\sigma, 0}(\bar{U}) \cap C^{0, \tau}(\bar{U}) .
\end{gathered}
$$

Les théorèmes 1.7 et 1.9 sont alors une conséquence des propositions 4.16 et 4.18 du fait que $p^{\prime} \leq p$ et de l'appartenance de $\sigma_{\alpha, n}$ à $\mathcal{S}^{\alpha_{1} / p+\alpha_{2} / p^{\prime}+2 m}$.

\section{Références bibliographiques}

[1] Agmon (S.), Douglis (A.) et Nirenberg (L.) . - Estimates near the boundary for solutions of elliptic partial differential equations satisfying general boundary conditions I. Comm. Pure Appl. Math. 17 (1964), pp. 35-92.

[2] Dauge (M.) .- Elliptic Boundary Value Problems on Corner Domains, Lecture Notes in Mathematics, Springer-Verlag 1341 (1988).

[3] Dauge (M.) et Steux (J.-L.) .- Problème de Dirichlet pour le laplacien dans un polygône curviligne, Journal of Differential Equations 70, $\mathrm{n}^{\circ} 1$ (1987), pp. 93-113.

[4] Grisvard (P.) .- Elliptic problems in non smooth domains, Pitman, London (1985).

[5] Grisvard (P.) .- Problèmes aux limites dans des domaines avec points de rebroussement, Ann. Fac. Sc. Toulouse II, $\mathbf{n}^{\circ} 3$ (1995), pp. 561-578.

[6] IвUKI (K.) .- Dirichlet problem for elliptic equations of second order in a singular domain of $\mathbb{R}^{2}$, J. Math. Kyoto Univ. 14, $\mathrm{n}^{\circ} 1$ (1974), pp. 55-71.

[7] Khelif (A.) .- Problèmes aux limites pour le laplacien dans un domaine à points cuspides, C. R. Acad. Sci. Paris 287. Série A. (1983), pp. 1113-1116, et Thèse présentée à l'Université de Nice (juin 1978). 
Problème de Dirichlet pour un opérateur elliptique dans un domaine à point cuspide

[8] Maz'ya (V. G.) et Plamenevskil (B. A.) .- Estimates in $L_{p}$ and in Hölder classes and the Miranda-Agmon Maximum Principe for solutions of Elliptic Boundary Value Problems in domains with singular Points on the Boundary, Amer. Math. Soc. Transl. 2 (1984), pp. 123-156; traduction de Math. Nachr. 81 (1978), pp. 25-82.

[9] Steux (J.-L.) .- Problème de Dirichlet pour le laplacien dans un domaine à point cuspide, C. R. Acad. Sci. Paris. 306, Série I (1988), pp. 773-776. 